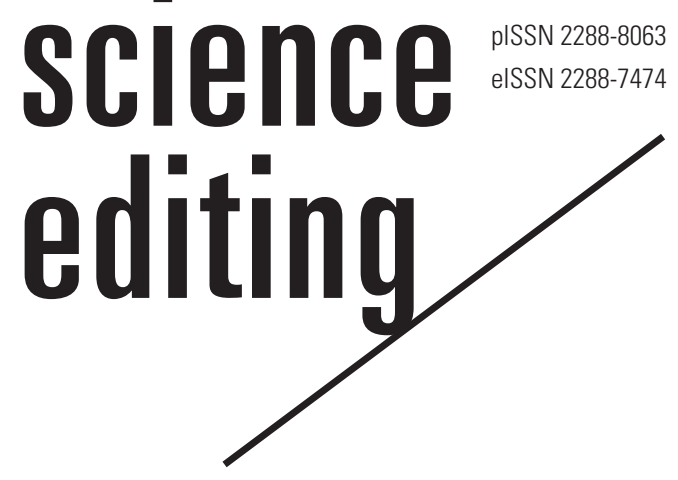

\title{
Report on the 2019 Workshop for Korea Manuscript Editors Certification Holders
}

\author{
Se Jueng Kim \\ MEDrang, Seoul, Korea
}

Date: July 12-13, 2019

Venue: Hyundai Bloomvista, Yangpyeong, Korea

Organizer: Korean Council of Science Editors

Three full years have already passed since I obtained a Korea Manuscript Editors Certification (KMEC), during which time I have never missed a single workshop for certification holders. At the two previous workshops, the issue of the most interest for journal editors from the past year was selected as the discussion topic, along with a separate dedicated time for sharing tips useful for the hands-on tasks of manuscript editor (ME). Initially, when looking forward to the topics that would be covered at this year's workshop of the Korean Council of Science Editors (KCSE), I expected that predatory journals or data sharing would again be the focus of attention. However, contrary to my expectations, this workshop primarily focused on practical aspects of business and provided valuable assistance regarding the challenges and questions that KMEC holders face in their day-to-day responsibilities. In this report, I will look back on my participation in the third workshop for KMEC holders held in Yangpyeong, Gyeonggi Province in July 2019, with the hope of conveying how beneficial the workshop was for various association members and publishing staff preparing to acquire a KMEC, as well as for KMEC holders who had not previously attended a KMEC workshop.

The Hyundai Bloomvista in Yangpyeong provided a venue for the workshop for KMEC holders from July 12 to 13,2019 . The workshop had changed quite a bit, both in terms of the venue and the topics, duration, and modalities of the lectures. On July 12, the first presentation started at 10 a.m., which was a little earlier than at the KMEC workshops held in earlier years. The presentation dealt with the definitions of supplements and appendices, as well as methods of processing and storing supplementary data when provided. In the past, many journals have

Received: January 13, 2020 Accepted: January 14, 2020

Correspondence to Se Jueng Kim 3jueng@medrang.co.kr

ORCID

Se Jueng Kim

https://orcid.org/0000-0001-8985-5205 suggested that authors avoid including supplements and appendices if possible, but as technology has advanced and the importance of data-sharing has become more widely recognized, it has become more common to attach extensive supplementary data when submitting manuscripts. MEs carrying out hands-on tasks have expressed concerns about processing these supplements. I learned that it is the role of the ME in such situations to assist the editorial board in establishing the journal's policies for handling these issues, and to ensure the consistency of all supplemental data processing methods. Above all, the presentation was easy to comprehend 

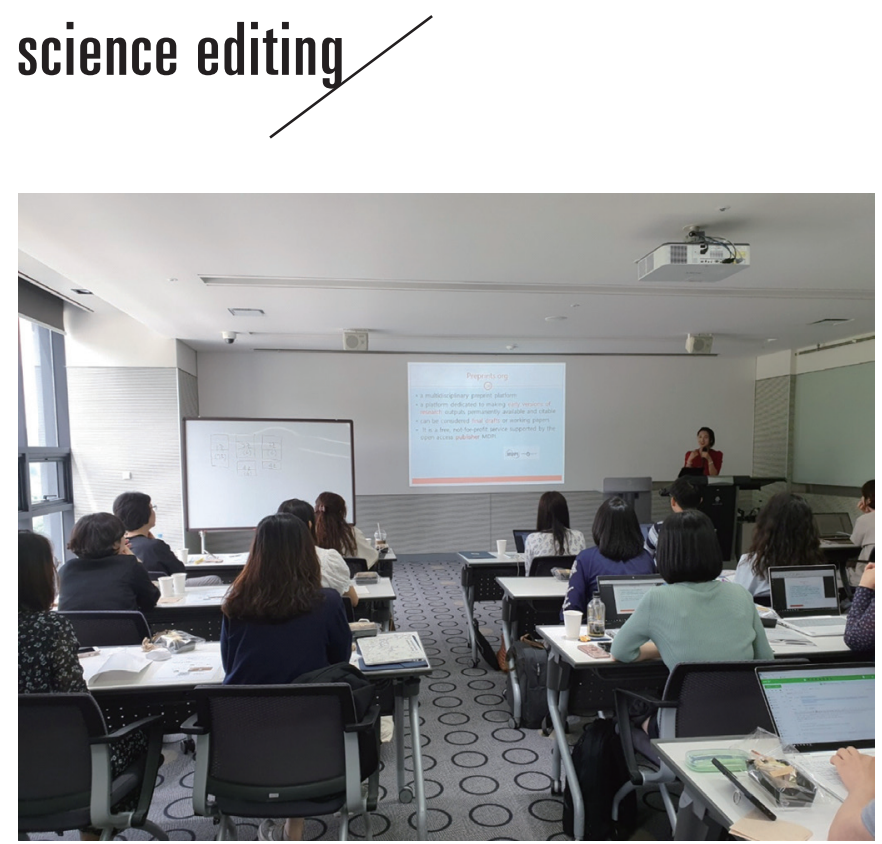

Fig. 1. Presentation during the Third Workshop for Korea Manuscript Editors Certification Holders (July 12-13, 2019).

because it used examples to show how supplementary data are handled by various journals.

The next lecture was on pre-prints (Fig. 1). To be honest, it was hard to understand the benefit of making materials available on public servers before they are submitted to journals or undergo peer review. One of my concerns would be that another researcher might write a paper based on wrong information if journals were to make manuscripts with critical errors available without screening, resulting in a scenario in which errors keep leading to errors. Nonetheless, pre-prints are expected to become increasingly common, given the fact that from another point of view, this rapid disclosure has advantages in terms of improving the feasibility of citations and allowing researchers to make claims on the ideas underlying the research. It seems that authors should make prudent decisions on pre-prints according to the prevailing standards in their field and the topic of their research.

After wrapping up the morning lectures, ginseng chicken soup (samgyetang) prepared for Chobok by the hotel was served for lunch, and the workshop resumed with the introduction of software useful for ME tasks and a brief demonstration.

As the scope of references is becoming more diverse, it is becoming more difficult to search for references, making it very time-consuming to proofread manuscripts. For this reason, my jaw dropped throughout this presentation. I was astonished to see a demonstration of a program that did not just automatically search for references, but was able to proofread them according to the journal's style-even though a final inspection by a human was naturally required to feel absolutely confident in the results. In addition, the workshop went on to introduce programs capable of correcting simple grammar and spelling errors when proofreading an English manuscript. Just as it is difficult to find Koreans who have perfect grammar and spelling in Korean, quite a lot of grammar, spelling, and punctuation errors are found in papers written by English-speaking authors. PerfectIt (Intelligent Editing Ltd., https://intelligentediting.com) and Grammarly (Grammarly Inc., https://www.grammarly.com) were presented as programs utilized in the revision of all manuscripts submitted in English, and the display of actual correction marks and how these programs could be applied within a manuscript was very helpful for understanding their function. It is expected that making good use of such programs when proofreading manuscripts will provide benefits in terms of both accuracy and speed. The programs that were presented in this session are currently proving to be useful for hands-on tasks, and anyone who feels out-of-date on new technologies (such as the present author) should feel free to obtain high-level information by participating in the next workshop.

The education session came to a close with a group exercise. Each group selected one academic journal and evaluated it according to the listing criteria for specific databases (Directory of Open Access Journals, Korean Federation of Science and Technology Societies, and Scopus) in order to determine the likelihood that the journal would be listed, and then practiced writing actual applications. Since the groups were assigned and participants were notified of their assignment by e-mail before the workshop, we chose the Journal of Obesity \& Metabolic Syndrome (JOMES) as the practice journal after a discussion among the group members in advance. At the workshop, the group members jointly reviewed whether the journal's homepage included all the corresponding information for every listing criterion, and the task was completed quickly due to the participation of MEs who worked for the selected practice journal, JOMES. In fact, JOMES is preparing to apply to be listed in Scopus this year. As a journal that meets all the criteria and has gained experience from submitting a previous application, there is no doubt that good results await.

The formal education program planned for the workshop concluded with presentations of each group's assessment. Although it is tempting to elaborate on the delightful dinner and debate that lasted until 11 p.m., as they were memorable events that should not be left out, I will instead try to avert the potential concern that it might sound like there were no opportunities to rest between the education and training sessions. In fact, sufficient breaks and nutritious meals were provided, along with time for the participants to become more closely acquainted with one another. Therefore, I urge any KMEC holders who might be hesitating to attend to put aside 
their worries and participate in this workshop.

Workshops for certificate holders are evolving every year. Although these workshops provide a venue for the participants to seek assistance, the KCSE staff who prepare the workshop always make the utmost effort to notice and meet participants' needs. It is definitely not an easy job to share their exclusive information related to hands-on tasks and to prepare for the presentations in addition to their regular work responsibilities, especially in a world where information is power and time is money. I would like to express my gratitude to the KCSE staff for taking their precious time to hold the workshop and would also like to gently express my heartfelt anticipation for the pleasant surprises that await in terms of the content that will be presented at next year's workshop.

\section{Conflict of Interest}

No potential conflict of interest relevant to this article was reported. 